\title{
Timing of renal replacement therapy in critically ill patients with acute kidney injury
}

\author{
Melanie Meersch ${ }^{1}$, Christoph Schmidt ${ }^{1}$, Joachim Schmidt ${ }^{2}$, Alexander Zarbock $^{1}$ \\ ${ }^{1}$ Department of Anesthesiology, Intensive Care and Pain Medicine, University of Münster, Münster, Germany; ${ }^{2}$ Department of Cardiac Surgery, \\ University of Münster, Münster, Germany \\ Correspondence to: Alexander Zarbock, MD. Department of Anesthesiology, Intensive Care and Pain Medicine, University of Münster, Albert- \\ Schweitzer-Campus 1, Gebäude A1, 48149 Münster, Germany. Email: zarbock@uni-muenster.de.
}

Submitted Aug 20, 2016. Accepted for publication Aug 24, 2016.

doi: 10.21037/atm.2016.09.05

View this article at: http://dx.doi.org/10.21037/atm.2016.09.05

The only available treatment option of severe acute kidney injury in critically ill patients is renal replacement therapy $(1,2)$. However, a number of issues affecting the optimal use of this primarily supportive technique still remain unresolved (3). The most important question now increasingly being asked is at what point and at which stage to initiate renal replacement therapy in critically ill patients with evolving acute kidney injury. Up to now, the KDIGO guidelines recommend to initiate renal replacement therapy emergently in case of the development of life-threatening complications such as hyperkalemia or severe fluid overload or greater imbalances or disruptions in homeostasis (2). The guidelines do not contain unequivocal instructions how to proceed when a progressive deterioration of kidney function coincides with any serious worsening of the patients' clinical condition, and leave the final decision for renal replacement therapy under these circumstances to the attending physician (2). The clear ambiguities of the KDIGO guidelines could erroneously be taken as a basis to delay renal replacement therapy in an attempt to give more time for spontaneous recovery of renal function. But anyway, mortality rates in patients requiring renal replacement therapy often exceed $50 \%$, suggesting that the optimal time point for initiation has not yet been identified (4).

In an effort to achieve deeper insights into the problems surrounding the initiation of renal replacement therapy, several small prospective trials have been conducted during the last years. Most of them demonstrate some beneficial effects of an early initiation of renal replacement therapy in critically ill patients with acute kidney injury (5-7). These observations were further underlined by a recently published meta-analysis highlighting better survival rates for patients, in whom renal replacement therapy was initiated early during the development of acute kidney injury (8). However, the conclusion was based on heterogeneous studies with ever varying definitions of what can be considered an "early" or a "late" initiation of renal replacement therapy. For these reasons it is hard to compare the results of the studies quoted above.

Two recent randomized controlled trials were more explicitly designed to specify the optimal timing for renal replacement therapy (the AKIKI and the ELAIN trials). These trials immediately revived the heated debate on when to start renal replacement therapy. In the study of Gaudry et al. (9) patients did not show a survival benefit at day 60 , whereas Zarbock et al. (10) demonstrated a significantly reduced 90 -day all-cause mortality with early initiation of renal replacement therapy. How is it that two studies addressing exactly the same problem reach such opposing conclusions? As discussed by Romagnoli et al. (11) and Honore et al. (12), differences in the respective study design might be crucial for the resolution of the conflict. The most important difference is the stage of AKI severity at which renal replacement therapy was initiated. In all patients enrolled in the AKIKI trial, acute kidney injury had already progressed to a very advanced stage (KDIGO stage 3 ). This degree of severity might already be too advanced for effective treatment with renal replacement therapy. This becomes apparent when comparing the mortality rates in the early group of the AKIKI trial and the late group of the ELAIN trial. These two groups included patients with almost identical severity stages (both KDIGO stage 3). Consequently, mortality rates of $48.5 \%$ and $54.7 \%$, respectively, were actually similar between the early AKIKI group and the late ELAIN group. Interestingly, 49\% 
of the patients in the delayed AKIKI group never received renal replacement therapy. Analyzing only those patients who received renal replacement therapy, mortality at 60 days was $48.5 \%$ in the early and $61.8 \%$ in the delayed AKIKI group. Those patients never receiving renal replacement therapy had lowest mortality (37.1\%), but were less ill at baseline compared to the other groups as shown by SOFA scores $(\mathrm{P}<0.0001)$.

Acute kidney injury is a systemic disease, which affects inflammation and function of different organs. It can be speculated that early treatment of acute kidney injury attenuates pro-inflammatory effects and a further decline in other organ function (13). This has recently been underlined by the fact that patients with early treatment showed a significant reduction of the pro-inflammatory cytokines Interleukin-6 and Interleukin-8, whereas patients with delayed treatment did not (10). Early initiation of renal replacement therapy may lead to clinical benefits by rapidly fixing metabolic and uremic derangements and controlling the harmful effects of fluid overload.

Of course, the indication for renal replacement therapy is always a balancing act between potential risks and benefits. Risk stratification concepts should therefore be taken in consideration. Exactly this is where the use of new AKI biomarkers must be introduced. These interesting molecules might be helpful in avoiding unnecessary therapy escalation in patients, who possibly do not need renal replacement therapy. However, it is important to measure biomarkers only in patients at a higher risk in order to improve their power for the prediction of progression of acute kidney injury and the need for renal replacement therapy (14).

In conclusion, both the AKIKI and the ELAIN trial provide new insights into the old issue of whether and to what extent early initiation of renal replacement therapy improves patients' outcome. The results of both studies are obviously not comparable due to high discrepancies in study design, and they both do not arrive at definitive conclusions. However, these findings together with the results of a recently performed meta-analysis (15) suggest that "early" initiation of renal replacement therapy might improve the survival of critically ill patients with acute kidney injury. Future multicenter randomized controlled studies are requested not to focus only on mortality but also on renal recovery and progression of acute kidney injury to chronic kidney disease.

\section{Acknowledgements}

Funding: This work was supported by the Else-Kröner-
Fresenius-Stiftung (2013_A46 to A.Z.).

\section{Footnote}

Provenance: This is a guest Correspondence commissioned by Section Editor Zhi Mao, MD (Department of Critical Care Medicine, Chinese People's Liberation Army General Hospital, Beijing, China).

Conflicts of Interest: M Meersch and A Zarbock received lecture fees from Astute Medical. And other authors have no conflicts of interest to declare.

Response to: Romagnoli S, Ricci Z. When to start a renal replacement therapy in AKI patients: many irons in the fire. Ann Transl Med 2016;4:355.

Honore PM, Jacobs R, Joannes-Boyau O, et al. Timing of renal replacement therapy in critically ill patients: where are the hands on the clock. Ann Transl Med 2016;4:354.

\section{References}

1. Bellomo R, Kellum JA, Ronco C. Acute kidney injury. Lancet 2012;380:756-66.

2. Wang C, Lv LS, Huang H, et al. Initiation Time of Renal Replacement Therapy on Patients with Acute Kidney Injury: A Systematic Review and Meta-analysis of 8179 participants. Nephrology (Carlton) 2016. [Epub ahead of print].

3. Ronco C, Ricci Z, De Backer D, et al. Renal replacement therapy in acute kidney injury: controversy and consensus. Crit Care 2015;19:146.

4. Hoste EA, Bagshaw SM, Bellomo R, et al. Epidemiology of acute kidney injury in critically ill patients: the multinational AKI-EPI study. Intensive Care Med 2015;41:1411-23.

5. Liu KD, Himmelfarb J, Paganini E, et al. Timing of initiation of dialysis in critically ill patients with acute kidney injury. Clin J Am Soc Nephrol 2006;1:915-9.

6. Gettings LG, Reynolds HN, Scalea T. Outcome in posttraumatic acute renal failure when continuous renal replacement therapy is applied early vs. late. Intensive Care Med 1999;25:805-13.

7. Shiao CC, Wu VC, Li WY, et al. Late initiation of renal replacement therapy is associated with worse outcomes in acute kidney injury after major abdominal surgery. Crit Care 2009;13:R171.

8. Karvellas CJ, Farhat MR, Sajjad I, et al. A comparison of early versus late initiation of renal replacement therapy in 
critically ill patients with acute kidney injury: a systematic review and meta-analysis. Crit Care 2011;15:R72.

9. Gaudry S, Hajage D, Schortgen F, et al. Initiation Strategies for Renal-Replacement Therapy in the Intensive Care Unit. N Engl J Med 2016;375:122-33.

10. Zarbock A, Kellum JA, Schmidt C, et al. Effect of Early vs Delayed Initiation of Renal Replacement Therapy on Mortality in Critically Ill Patients With Acute Kidney Injury: The ELAIN Randomized Clinical Trial. JAMA 2016;315:2190-9.

11. Romagnoli S, Ricci Z. When to start a renal replacement therapy in AKI patients: many irons in the fire. Ann Transl Med 2016;4:355.

12. Honore PM, Jacobs R, Joannes-Boyau O, et al. Timing

Cite this article as: Meersch M, Schmidt C, Schmidt J, Zarbock A. Timing of renal replacement therapy in critically ill patients with acute kidney injury. Ann Transl Med 2016;4(18):360. doi: 10.21037/atm.2016.09.05 of renal replacement therapy in critically ill patients: where are the hands on the clock. Ann Transl Med 20162016;4:360.

13. Pohl J, Papathanasiou M, Heisler M, et al. Renal replacement therapy neutralizes elevated MIF levels in septic shock. J Intensive Care 2016;4:39.

14. Goldstein SL, Chawla LS. Renal angina. Clin J Am Soc Nephrol 2010;5:943-9.

15. Wang C, Lv LS, Huang H, et al. Initiation Time of Renal Replacement Therapy on Patients with Acute Kidney Injury: A Systematic Review and Meta-analysis of 8179 participants. Nephrology (Carlton) 2016. [Epub ahead of print]. 